\title{
Criminologie
}

\section{Perspectives traditionnelles et perspectives critiques en criminologie}

\section{Marie-Andrée Bertrand}

Volume 19, numéro 1, 1986

Politiques et pratiques pénales. 25 ans de réflexion et d'action

URI : https://id.erudit.org/iderudit/017228ar

DOI : https://doi.org/10.7202/017228ar

Aller au sommaire du numéro

\section{Éditeur(s)}

Les Presses de l'Université de Montréal

ISSN

0316-0041 (imprimé)

1492-1367 (numérique)

Découvrir la revue

Citer cet article

Bertrand, M.-A. (1986). Perspectives traditionnelles et perspectives critiques en criminologie. Criminologie, 19(1), 97-111. https://doi.org/10.7202/017228ar
Résumé de l'article

Is critical criminology "passée"? Have its fathers, the British and American sociologists who wrote Critical Criminology in the mid seventies exhaust its potential interest and flavour?

It would be too bad because critical criminology never really took place. There never was a serious and rigorous attempt at unfolding the historical, epistemological, socio-political roots of the discipline, a critical look at it that took nothing for granted.

Reminding the readers of the very serious and highly publicised debate around Traditional and Critical Theory in the late thirties launched by the sociologists and philosophers of the Frankfurt School, the author shows that, far from being outdated, critical theory is of the utmost practicality in criminology, even more so because its founding fathers have taken, since, a less partisan and doctrinaire view of it. The applications of their intellectual and socio-political orientations to criminology are numerous, calling for a serious socio-historical analysis of the discipline and of its academic origins that should throw light on where it is going and its impotence at developing a paradigm.
Tous droits réservés @ Les Presses de l'Université de Montréal, 1986
Ce document est protégé par la loi sur le droit d'auteur. L’utilisation des services d’Érudit (y compris la reproduction) est assujettie à sa politique d'utilisation que vous pouvez consulter en ligne.

https://apropos.erudit.org/fr/usagers/politique-dutilisation/ 


\section{PERSPECTIVES TRADITIONNELLES ET PERSPECTIVES CRITIQUES EN CRIMINOLOGIE}

Marie-Andrée Bertrand*

Is critical criminology «passee»? Have its fathers, the British and American sociologists who wrote Critical Criminology in the mid seventies exhaust its potential interest and flavour?

It would be too bad because critical criminology never really took place. There never was a serious and rigorous attempt at unfolding the historical, epistemological, socio-political roots of the discipline, a critical look at it that took nothing for granted.

Reminding the readers of the very serious and highly publicised debate around Traditional and Critical Theory in the late thirties launched by the sociologists and philosophers of the Frankfurt School, the author shows that, far from being outdated, critical theory is of the utmost practicality in criminology, even more so because its founding fathers have taken, since, a less partisan and doctrinaire view of it. The applications of their intellectual and socio-political orientations to criminology are numerous, calling for a serious socio-historical analysis of the discipline and of its academic origins that should throw light on where it is going and its impotence at developing a paradigm.

\section{INTRODUCTION}

Les collègues à qui je me suis ouverte de mon intention d'écrire sur le sujet des perspectives traditionnelles et perspectives critiques en criminologie m'ont accueillie avec surprise, ennui ou inquiétude.

«Qui a-t-il de nouveau sur ce front ?» semblaient dire certains.

«N'a-t-on pas assez parlé de criminologie critique sans que cela renouvelle la thérie et surtout sans que cela enrichisse la pratique criminologique? Faut-il vraiment y revenir?» objectait carrément un confrère.

\footnotetext{
* Professeure titulaire à l'École de criminologie de l'Université de Montréal. Mes remerciements vont aux personnes suivantes, qui ont bien voulu lire les premières versions de ce texte et me proposer diverses corrections : Maryse Barbance, Jacqueline De Plaen, Daniel Élie, Francyne Goyer, Jean Trépanier.
} 
«Que vas-tu loger à l'enseigne de la perspective traditionelle?» s'inquiétait un autre.

«Tu veux faire un article d'inspiration marxiste?» suggérait un professeur familier avec l'École de Francfort et sachant par ailleurs que ma culture marxienne est de niveau sous-gradué, il s'apprêtait à me décourager de toute tentative en ce sens. «La théorie critique en sciences sociales est marquée par les marxistes. Comment te sortiras-tu des confusions que tu auras créées par l'emploi d'un vocabulaire déjà investi?»

Si j'ai persisté dans mon projet, c'est, en partie, parce qu'il m'apparaissait que la théorie critique et ses perspectives méritent d'être dédouanées, de retrouver une certaine virginité.

Ce ne sont pas les marxistes qui ont inventé la critique, ils l'ont investie d'un sens particulier à une époque bien précise. Bien avant eux, un certain philosophe nommé Kant s'était d'ailleurs presque approprié le terme dans un sens fort différent... Ce ne sont pas non plus les criminologues britanniques qui ont inventé la criminologie critique, à supposer qu'il existe un courant identifiable qui mérite ce nom. La paternité d'une critique économicosociale des "causes» de la criminalité reviendrait plutôt à un Hollandais du nom de Bonger (1905) ! Si j'ai persisté dans mon projet, c'est aussi pour exposer les avantages qu'il y aurait à nous situer plus souvent dans une ou des perspectives critiques.

La critique dont je veux parler ici s'intéresse à l'épistémè. Essentiellement, elle propose de ne rien prendre pour acquis, surtout pas notre objet, et de nous efforcer, dans toute entreprise intellectuelle, de reconnaître les mobiles qui nous poussent dans une direction plutôt que dans une autre; de nous interroger sur ce qui fait se concentrer la science sur certains objets plutôt que sur d'autres.

Puisque ma proposition critique veut se démarquer de la Théorie critique née à l'École de Francfort, elle ne peut en faire abstraction : les philosophes allemands de l'Institut de recherche sociale et de l'École de Francfort ont opposé fermement théorie traditionnelle et théorie critique, les décrivant comme irréconciliables. Mais que faut-il penser de cette opposition? Ne convient-il pas de rappeler l'histoire de cette antinomie et de revoir ce qui amenait Adorno, Fromm, Habermas, Horkheimer et Marcuse à s'élever comme ils l'ont fait contre la science traditionnelle? Dans 
ces travaux marquants qu'ils ont conçus avant même d'émigrer aux États-Unis, fuyant le national-socialisme, qu'avaient-ils à reprocher à la théorie conçue de façon traditionnelle?

\section{«THÉORIE TRADITIONNELLE ET THÉORIE CRITIQUE»} 1937

C'est ainsi que s'intitule le manifeste dans lequel, en 1937, Max Horkheimer a campé la théorie traditionnelle et la théorie critique dans des positions extrêmement contrastées. La théorie traditionnelle remonte, selon lui, aux origines de la philosophie moderne avec Descartes qui pose comme règle de la méthode scientifique qu'il faut «conduire par ordre ses pensées en commençant par les objets les plus simples et les plus aisés à connaître pour monter peu à peu comme par degrés jusques à la connaissance des plus composés» (Discours de la méthode, II). Selon Horkheimer, les sciences de l'homme ont copié les sciences de la nature dans leur méthode mais ont oublié de réfléchir sur leur objet qui est aussi sujet :

La patiente et minutieuse collecte de l'information dans tous les domaines scientifiques qui ont pour obiet la vie sociale, l'accumulation d'immenses quantités de données fragmentaires concernant ses problèmes, les recherches empiriques menées aux moyens d'enquêtes consciencieuses ou d'autres expédients : tout cela qui, depuis Spencer, constitue en particulier dans les pays anglo-saxons une grande partie de l'activité universitaire, offre certes un spectacle apparemment plus proche de l'ensemble de la vie dans la civilisation industrielle que le travail de cabinet qui a caractérisé, par exemple, une partie de la sociologie allemande, la formulation de principes abstraits. la réflexion théorique sur les concepts fondamentaux. Mais il ne faut voir là nulle différence dans la structure de la pensée. (Horkheimer, 1937, 18)

Pour l'auteur de "Théorie traditionnelle et Théorie critique», les partisans de la voie traditionnelle, qu'ils soient des empiristes ou des théoriciens partagent une conception identique de la connaissance à cette différence près que les empiristes refusent le travail sur les principes généraux :

Les empiristes ne se représentent pas la théorie, poussée jusqu'au bout de son élaboration, autrement que les théoriciens... Ils sont simplement imbus de la conviction quelque peu suffisante qu'étant donné la complexité des problèmes sociologiques et l'état actuel des connaissances, le travail sur 
les principes généraux peut être considéré comme une occupation aussi commode qu'oiseuse. (Ibid., 19)

L'activité coupée du réel qui se pratique dans certains secteurs de l'entreprise universitaire, l'intelligence qui se dépense en pure perte dans la construction d'idéologies : tout cela, qui concourt à l'existence de la société telle qu'elle est, est nécessairement récupéré par les forces sociales en place (ibid., 37).

Mais que serait donc la théorie critique? Ou plutôt qu'était la théorie critique comme l'envisageait Horkheimer en 1937? D'abord une capacité de prendre une distance par rapport aux règles dominant la vie quotidienne alors que la majorité des gens pensent qu'il suffit d'accepter le sort qui leur est fait, les déterminations auxquelles leur existence est soumise et qu'ils trouveront leur dignité à accomplir leurs tâches telles que prescrites, Horkheimer croit qu'il faut plutôt adopter une attitude de critique caractérisée «par une méfiance totale à l'égard des normes de conduite que la vie sociale telle qu'elle est organisée fournit à l'individu" (ibid, 38)

L'auteur contraste dans des termes précis la pensée théorique traditionnelle et la pensée critique : la première oppose valeur et recherche, savoir et agir, et évite ainsi au savant d'éprouver les contradictions inévitables entre son travail et son cadre de pensée; la seconde, au contraire, fait porter l'effort de la pensée à se connaitre elle-même afin que se révèlent les rapports entre les positions intellectuelles et l'appartenance sociale (ibid., 40).

Mais ne faut-il pas faire la critique de la théorie critique qui : apparaît comme entachée de subjectivité, spéculative, unilatérale et inutile et «donne une impression de partialité et d'injustice... parce qu'elle va contre les habitudes de pensée en vigueur qui contribuent à perpétrer le passé» (ibid., 51).

La Théorie critique. Horkheimer ne manque pas de le signaler, ne peut se prévaloir d'aucune réalisation concrète. Bien sûr, le nombre de ses partisans s'est accru après 1937; l'École de Francfort a eu une influence réelle et certains de ses adhérents ont occupé des positions de force dans les partis politiques. Mais un certain niveau de succès n'a pas été atteint. Et certaines performances, Horkheimer l'avoue, se révèlent ou se révéleront de fausses victoires ou des erreurs (ibid., 52). 
Dans un article de 1970: «La Théorie critique, hier et aujourd'hui» (in Théorie critique (1978), p. 353-369), Horkheimer revient sur ses propos de 1937 avec une sagesse et une maturité émouvantes. Il fait d'abord la critique de son enthousiasme d'alors pour le marxisme et surtout de son attitude révolutionnaire générée par le national-socialisme des années 1930 . Il reconnaît que la théorie critique qu'il prônait quarante ans plus tôt était en partie une réaction au national-socialisme : la société d'alors était devenue par le national-socialisme plus injuste qu'auparavant et seule une société meilleure pouvait instaurer les conditions d'une pensée vraie... Marx avait tort sur de nombreux points, ajoute Horkheimer en 1970 : la paupérisation croissante de la classe ouvrière, prédite par l'auteur du Capital ne s'est pas produite. On est face à des sociétés où le nombre des employés grandit et dépasse de beaucoup celui des travailleurs manuels. Les crises économiques sont devenues plus rares. Enfin, il faut bien le reconnaître, plus de justice n'égale pas plus de liberté. Au contraire, plus il y a de justice, moins il y a de liberté. Et, pronostic pessimiste : «Nous sommes convaincus que la société va évoluer finalement vers un monde totalement administré»(Horkheimer, 1978, 359).

Dans ce manifeste deuxième manière, cette espèce de version corrigée de sa déclaration de foi de 1937 en la théorie critique, Horkheimer, touché par la mort d'Adorno qui devait être présent à la conférence où il présentait sa communication sur la nouvelle théorie critique, parle de son vieil ami, parle aussi de la religion et de la fonction qu'on lui nie dans les sociétés contemporaines et finalement revient sur la fonction de la théorie critique :

elle doit attirer l'attention sur le prix du progrès, sur ce danger qu'il entraîne avec lui jusqu'à la disparition de l'idée du sujet autonome, de l'idée de l'âme... (ibid., 1978, 364)

À la toute fin de cette communication, Horkheimer rappelle la fonction de l'université : mettre de l'ordre dans la science, la transmettre complètement sinon celle-ci disparaîtra et l'homme n'y attachera aucun intérêt. Fidèle à sa conviction critique. il rappelle que le grand devoir des professeurs d'université c'est d'immuniser les étudiants contre la séduction démagogique et suggère que les activistes sont bien près des démagogues. Aussi faut-il dénoncer les demi-vérités qu'ils charrient dans leur pratique. 


\section{PERSPECTIVES TRADITIONNELLES ET PERSPECTIVES CRITIQUES EN CRIMINOLOGIE AUJOURD'HUI}

La première section de cet article, consacrée à deux œuvres d'un sociologue de l'École de Francfort, voulait rappeler quel contraste, quelle antinomie une certaine sociologie radicale des années 30 a voulu voir entre ce qu'elle appelait la théorie traditionnelle et la théorie critique. Horkheimer n'est pas le seul à avoir vu ces oppositions et à avoir prôné une "conscience» de l'activité intellectuelle par opposition à une science neutre. Mentionnons Adorno, Frenkel-Brunswick, Levinson et Sandford (1950), Althusser $(1969,1970$ et 1976), Becker et Horowitz (1972), Bottomore (1964 et 1968), Dahrendorf (1959), Goodman (1970), Gouldner (1970), Gramsai (1971), Habermas (1975 et 1976), Horowitz (1969), Kolko (1969), Lefebvre (1958 et 1975), Lukas (1971), Lynd (1969), Mandel (1968 et 1971), Marcuse (1962, 1968 et 1969), Mills (1956, 1959, 1960, 1962 et 1964), Wellmer (1974), Zeitlin (1967 et 1968), etc. Voilà quelques-uns de ceux parmi les philosophes sociaux et les sociologues qui ont exigé que la science s'adonne à un examen des orientations de la pensée, à l'analyse des choix de ses cibles d'étude.

Éveillée pendant mes études de doctorat à quelques-unes des propositions de la pensée critique et d'une certaine perspective radicale par les professeurs et les étudiants gradués de l'École de criminologie de l'Université de Californie à Berkeley (1965-67) et du Center for the Study of Law and Society de la même université, stimulée par les écrits de quelques-uns d'entre eux (Bittner et Platt, 1966) puis par les travaux du National Deviancy Conference, en Angleterre, qui devait donner naissance au European Group for the Study of Deviance and Social Control et donc à The New Criminology (1973) et à Critical Criminology (1975), j'entreprenais en 1977, avec F. Acosta et P. Sohet de recencer les œuvres de nos collègues qui s'intéressaient à la théorie criminologique dans une perspective critique.

À cette époque, il y a maintenant tout près de dix ans, notre collecte était déjà intéressante. On retrouvait les travaux de Bianchi, Simondi et Taylor (1975), Bittner et Platt (1966), Brady (1975), Brodeur (1977), Christie (1968 et 1974), Ciacci et Simondi (1977), Cohen (1973), Dahl (1977), Dandurand (1975), Faugeron (1975), Feest, Huchting et Lange (1975), Klein et Kress (1976), Lascoumes (1975 et 1976), Mathiesen (1974), 
Melossi (1975, 1976 et 1977), Miaille (1976), Peraldi (1976), Pires (1975), Platt (1970 et 1974), Quinney (1972 et 1974), Robert (1972 et 1976), Schwendinger et Schwendinger (1970, 1972, 1974 et 1977), Takagi (1974), Taylor et Taylor (1973), Taylor, Walton et Young (1973 et 1975), Turk (1975), Van Outrive (1973 et 1977), etc.

Depuis, d'autres inventaires et, fort heureusement, des critiques de la critique ont été élaborés notamment par Baratta (1982 et 1983), Brodeur (1984), Hulsman (1982) et Robert (1984) rappelant, analysant ou prenant le contrepied de travaux américains, italiens, québécois, français, hollandais, etc.

Qu'il s'agisse d'auteurs ayant proposé des positions critiques, c'est-à-dire "réflectives», visant à développer une conscience des mobiles qui guident le choix de nos sujets d'étude et la mise au clair de l'identité des sujets et des auteurs, ou de commentateurs critiques qui s'intéressent aux essais des théoriciens critiques pour en indiquer les limites et se situer dans des perspectives connexes ou différentes ou, enfin, d'auteurs qui font la critique à partir d'une doctrine socio-économique comme le marxisme, il me semble que, pour l'instant, la distinction entre tous ceux-là n'est pas immédiatement importante. Ce que j'ai voulu souligner, c'est que tant chez les sociologues, chez les philosophes sociaux que chez les criminologues, depuis quarante ans ou vingt ans, selon le cas, un mouvement important, des énergies considérables se sont concentrés autour de ce que l'on peut appeler, de façon large, une réflexion critique.

\section{DE QUELLES PERSPECTIVES CRITIQUES S'AGIT-IL?}

Le lecteur aura remarqué qu'à la différence de Max Horkheimer je n'ai pas parlé, dans le titre de mon article, de théorie traditionnelle et de théorie critique, mais de Perspectives traditionnelles et perspectives critiques en criminologie. Le choix des mots et du pluriel est important. Commençons par le choix des mots : perspectives, plutôt que théorie. Dans l'état actuel d'avancement des connaissances dans le domaine précis qui est le nôtre, les orientations "traditionnelles» plutôt que "critiques» sont des perspectives plutôt que des positions théoriques. Le pluriel, car nous croyons aussi qu'il serait trop simple de prétendre qu'il y a unicité de perspective chez les «traditionalistes» et par opposition aux «critiques», en deux camps bien départagés et 
clivés. Certes, il existe parmi les sociologues de la déviance et parmi les criminologues de profession et de carrière des empiristes «durs» et même des théoriciens «durs» qui soutiennent que la neutralité scientifique est la seule position tenable tout comme on trouve des radicaux et des "critiques», qui méprisent l'empirie ou même la théorie fondée sur l'induction traditionnelle à postulat de «neutralité». Mais nous croyons que ces extrêmes sont minoritaires. Mais peut-on pratiquer la science «traditionnelle» avec des vues critiques?

Disons d'abord que Horkheimer lui-même avait bien arrondi les angles dans son article de 1970. La maturité et sans doute l'éloignement temporel et géographique de la menace du nationalsocialisme allemand lui avaient fait replacer, comme on l'a vu, au centre de ses préoccupations l'autonomie de la personne et sa liberté. Disons ensuite que la maturation qu'ont connue les sciences sociales et spécialement la criminologie québécoise depuis 25 ans a permis, nous semble-t-il, un certain rapprochement de deux perspectives.

Plusieurs criminologues d'orientation traditionnelle, c'est-àdire pratiquant l'empirie avec rigueur ou se mouvant dans la théorie selon les canons habituels de l'analyse des idées ont développé au fur et à mesure de leur maturation intellectuelle, de leurs intérêts personnels ou des chocs sociaux et politiques qu'ils vivent, des préoccupations de critique sociale, d'analyse personnelle de leurs mobiles, et soit par le chemin de l'épistémologie, soit par la voie historique, soit par l'engagement politique et moral, soit suite à une analyse personnelle leur ayant apporté les bénéfices d'un éclairage considérable sur leurs motivations et leur cheminement, en arrivent, chacun à sa façon, à identifier les conflits sociaux qui opposent les groupes humains dans des rapports inégaux, à dénoncer la nature servile des rapports des intellectuels prétendument neutres avec les hommes de pouvoir et à retrouver ainsi une distance et une liberté qui sont les garanties d'une saisie «intelligente» au sens étymologique, des phénomènes.

\section{UN PROGRAMME DE TRAVAIL}

Il me semble que la théorie critique criminologique, la critique de la criminologie et les perspectives radicales en criminologie ont cependant négligé trois sources d'aveuglement intellectuel ou 
affectif sur notre objet et sur nos démarches. Je les énumère ici plutôt comme des hypothèses ou des questions.

Premièrement, les déterminismes sociaux, spatio-temporels de la naissance de la criminologie québécoise ne me paraissent pas avoir été explorés dans leur signification et leur impact sur les rapports que cette discipline entretient avec les différents organes de pouvoir judiciaire, policier, économique, politique. Certes, nous disposons de quelques chroniques des origines de l'École de criminologie de l'Université de Montréal, mais celles-ci, y compris celle à laquelle j'ai collaboré (Normandeau et Bertrand, 1984), ne font pas l'histoire de la criminologie et ne rendent pas compte des forces en présence : conflit entre le psychologique et le sociologique, inconscience d'une profession naissante qui s'apprête à servir des structures pénales dont elle n'a pas bien analysé les orientations et la place dans l'organisation socio-politique. Comme il serait important de bien connaître les facteurs qui ont présidé à la professionnalisation de cette école de criminologie qui est la nôtre! Quels intérêts étaient en jeu? Quels étaient les vrais mobiles des auteurs de la mise au monde de la criminologie québécoise? - Sans doute faut-il rendre ici hommage au fondateur de l'École qui, dans un article publié dans cette revue même, un long article autobiographique (Szabo, 1977), nous fait part de son cheminement de ses contacts et identifie quelques acteurs.

Mais on doit maintenant poursuivre l'analyse socio-historique de cette institution, des conflits particuliers qui en ont constitué la dynamique de départ, conflits surgissant de la divergence des interêts personnels et structurels, politiques et scientifiques, tout cela qui a marqué profondément l'orientation des programmes et le choix des collaborateurs.

Deuxièmement, il nous faudrait faire pour nous-mêmes et en l'appliquant au cas particulier de la criminologie québécoise et canadienne, l'histoire universitaire canadienne et québécoise. Ici encore, il faut remercier Denis Szabo qui a fait écrire plus d'une page de l'histoire des sciences sociales au Québec, dans Continuités et Ruptures (1984), travail à poursuivre dans le cadre plus large de l'histoire des universités franco-québécoises et donc des origines de la criminologie dans le milieu universitaire; l'étude des conséquences de la localisation de la criminologie à Montréal, de sa séparation d'avec la sociologie autant de facteurs qui déterminaient ses clientèles et ses enseignements. 
L'histoire du développement des institutions universitaires canadiennes et québécoises, de l'explosion de leurs clientèles, de la provenance et des aspirations de celles-ci; de sa génération quasi spontanée de la criminologie dans les années soixante puis de la spécialisation; des lieux de sa formation - tout cela nous aidera à mieux voir d'où viennent nos écoles et départements de criminologie au Canada, quel chantage à la clientèle les responsables politiques et les administrateurs universitaires ont exercé sur les directeurs des écoles de criminologie en même temps que les milieux de pratique proposaient ou imposaient leurs définition du succès du traitement et quelles sont les conséquences de ces contrôles sur les programmes d'étude et de recherche.

Troisièmement, il nous faut aussi faire l'analyse du «sexe» des enseignements et des recherches qui ont pris place dans cette institution, un sexe qui peut avoir peu de choses à voir avec celui des enseignants et des chercheurs. L'École a tôt attiré des étudiantes, des chercheures et des professeures féminines. Cela ne signifie en rien que sa science n'était pas profondément sexiste et que l'influence que l'École a pu avoir sur les institutions pénales était de quelque façon débarrassée de biais de ce "genre». Ce troisième décapage est entrepris dans la criminologie américaine, française, québécoise et britannique, pour ne mentionner que les sources qui nous sont le plus familières.

Ce sont là des jalons de réflexion, d'étude, de recherche, et peut-être des motifs d'engagement intellectuel et social. Les exigences de la connaissance et d'une action historiquement consciente passent par ces examens minimaux.

Parlant de la nécessité de revoir de façon critique l'histoire de notre École, des agents qui en ont été les créateurs, de ceux qui en ont été les coconstructeurs, on pense ici à l'utilité d'une psychanalyse de nos origines ou d'une socianalyse ou les deux, qui permettra de retrouver le sens d'un ensemble biographique devenu impénétrable aux sujets eux-mêmes. On le sait, dans la biographie actualisée, la mémoire fait défaut à tel point que les troubles de cette faculté exigent eux-mêmes le recours à l'herméneutique.

Tous les recours contre la fausse conscience nous aideront à faire de l'entreprise d'enseignement et de recherche en criminologie une œuvre plus éclairée et surtout plus éclairante pour nos étudiants et pour les lecteurs de nos travaux. Qu'il s'agisse de travaux 
historiques comme en a commencé le Groupe d'étude qui a déjà produit sur l'histoire du service social un décapage absolument remarquable (voir Groulx : le Service social confessionnel au Canada français, 1983); qu'il s'agisse de l'histoire des institutions pénales ou d'une réflexion sur nos enseignements, sur l'orientation de nos recherches; qu'il s'agisse d'entreprises recourant davantage à la philologie, à l'épistémologie, à la critique de l'économie politique, à la psychanalyse, etc., tous les angles, toutes les perspectives qui commandent l'interrogation la plus large possible de notre savoir et de ses orientations doivent être utilisés au service d'une connaissance et d'une théorie dignes de ce nom.

Mais, me demandent certains, à quoi servira d'avoir les réponses à ces questions? Que ferons-nous de ces réponses? - Il me semble que la connaissance de nos origines nous permettra de savoir qui nous sommes et où nous pouvons aller, ce que nous pouvions mieux faire et ce à quoi il faut renoncer. Elle éclairerait d'un jour nouveau la remarque d'un autre collègue s'inquiétant de l'absence de paradigme ou de l'impuissance des paradigmes connus à expliquer et surtout à prédire, en criminologie. Cette fois, c'est l'objet même de la discipline que nous sommes invités à reconsidérer.

\section{BIBLIOGRAPHIE}

ACOSTA, F., M.A. BERTRAND et P. SOHET (1977), les Différentes orientations de la criminologie nouvelle, Rapport de recherche, Université de Montréal, École de criminologie, $84 \mathrm{p}$.

ADORNO, T.W., E. FRENKEL-BRUNSWICK, D.J. LEVINSON et R.N. SANFORD (1950), The Authoritarian Personality, New York, Harper \& Row.

ALTHUSSER, L. (1969), For Marx, Translated by B. Brewster, London, Allen Lane.

ALTHUSSER, L. (1976), Positions (1964-75), Paris, Éditions sociales.

ALTHUSSER, L. et E. BALIBAR (1970), Reading «Capital», Translated by B. Brewster, London, NLB Press.

BARATTA, A. (1982), "Conflit social et criminalité, Pour la critique de la théorie du conflit en criminologie», Déviance et Société, 6, 1, 1-23.

BARATTA, A. (1983), «Criminologie critique et critique du droit pénal; introduction à la sociologie juridico-pénale», Cahier no 14, Université de Montréal, École de criminologie, $230 \mathrm{p}$.

BECCARIA, C. (1979) (1764), Des délits et des peines, Paris, Flammarion. 
BECKER, H.S. et I.L. HOROWITZ (1972), «Radical politics and sociological research : observations on methodology and ideology», American Journal of Sociology, 78, juillet, 48-66.

BIANCHI, H., M. SIMONDI, I. TAYLOR (édit.) (1975), Deviance and Control in Europe, London, John Wiley and Sons.

BITTNER, E. et A.M. PLATT (1966), «The meaning of punishment», Issues in Criminology, 2, (1), 79-99.

BONGER, W. (1905), Criminalité et conditions économiques, Amsterdam, Tierie.

BOTTOMORE, T.B. (1964), «Preface», pp. v-vii, in Karl Marx, Selected Writings in Sociology and Social Philosophy, Edited and Translated by T.B. Bottomore, New York, McGraw-Hill.

BOTTOMORE, T.B. (1968), Critics of Sociefy : Radical Thought in North America, New York, Pantheon.

BRADY, J.P. (1975), "The talking stone: evolution and action of people's criminology», The Insurgent Sociologist, V, (IV), 73-79.

BRODEUR, I.P. (1977), Quelques remarques sur la criminalité radicale, Travail présenté dans le cadre du Séminaire de criminologie radicale, Université de Montréal, École de criminologie.

BRODEUR, J.P. (1984), «La criminologie marxiste : controverses récentes», Déviance et Société, 8, 1, 43-70.

CHRISTIE, N. (1968), "Changes in penal values», in Scandinavian Studies in Criminology, vol. 11, 161-172, London, Tavistock Publications.

CHRISTIE, N. (édit.) (1974) . Scandinavian Studies in Criminology, vol. V, London, Martin Robertson.

CIACCI, M. et M. SIMONDI (1977), «Un courant novateur du savoir criminologique : l'expérience de l'European Group of the Study of Deviance and Social Control, Déviance et Société, 1, (1), 109-117.

COHEN, S. (1972), Moral panics and Folk devils, London, MacGibbon and Kee.

DAHL, T.S. (1977), «State intervention and social control in nineteenthcentury Europe», Contemporary Crises, 1. (2), 163-187.

DAHRENDORF, R. (1959), Class and Class Conflict in Industrial Society, Stanford, Calif., Stanford University Press.

DANDURAND, Y. (1975), "Radical criminology in Canada», Crime and Social Iustice, 4, 49.

FAUGERON, Claude et al. (1975), De la déviance et du contrôle social, Recherche réalisée avec l'aide de la Délégation générale à la recherche scientifique et technique (France).

FEEST, J., K. HUCHTING et W. LANGE (1975), The Changing Nature of Legal Repression - Report on West Germany, Third Conference of European Group for the Study of Deviance and Social Control, Amsterdam, 9.12 septembre.

FROMM, E. (1941), Escape from Freedom, New York, Holt.

FROMM, E. (1955), The Sane Society, Greenwich, Conn., Fawcett Publications Inc.

GOODMAN, M. (1970), The Movement Toward a New America. New York, Knopf.

GOULDNER, A.W. (1970), The Coming Crisis of Western Sociology, New York, Basic Books. 
GRAMSCI, A. (1971), Selections from the Prison Notebooks, Edited and translated by Quintin Hoare and Goeffrey Nowell Smith, New York, International Publishers.

GROULX, L. (1983), «Le service social confessionnel au Canadafrançais», Revue canadienne de service social, 83, 141-161.

HABERMAS, I. (1975), Legitimation Crisis, Translated by Thomas McCarthy, Boston, Beacon Press.

HABERMAS, J. (1976), Connaissance et intérêt. Traduit de l'allemand par G. Clémençon, Paris, Les Éditions Gallimard.

HORKHEIMER, M. (1937), Théorie traditionnelle et théorie critique, Paris, Les Éditions Gallimard.

HORKHEIMER, M. (1978), Théorie critique, Paris, Payot.

HOROWITZ, D. (1969), Empire and Revolution : A Radical Interpretation of Coniemporary History, New York, Random House.

HULSMAN, L. et J. BERNAT DE CELIS (1982), Peines perdues. Le système pénal en question, Paris, Éditions du Centurion.

KLEIN, D. et J. KRESS (1976), «Any woman's blues : a critical overview of women, crime, and the criminal justice system», Crime and Social Justice, 5, 34-49.

KOLKO, G. (1969), The Roots of American Foreign Policy; An Analysis of Power and Purpose, Boston, Beacon Press.

LASCOUMES, P. (1975), «Criminologie : savoir et ordre», Actes, 9, 5-12.

LASCOUMES, P. (1976), «La criminologie en action ou l'ordre rationalisé», Actes, 11, 33-40.

LEFÈBVRE, H. (1958), Critique de la vie quotidienne, Paris, L'Arche.

LEFĖBVRE, H. (1975), Hegel - Marx - Nietzsche ou le Royaume des Ombres, Tournai, Belgique, Les Éditions Casterman.

LUKACS, G. (1971), History and Class Consciousness : Studies in Marxist Dialectics, Translated by Rodney Livingstone, Cambridge, Mass., MIT Press.

LYND, S. (1969), The New Left, Boston, Porter Sargent.

MANDEL, E. (1968), Marxist Economic Theory, 2 vol., Translated by Brian Pearce, New York, Monthly Review Press.

MANDEL, E. (1971), «Workers and permanent revolution», pp. 169-187, in George Fischer (édit.), The Revival of American Socialism: Selected Papers of the Socialist Scholars' Conference, New York, Oxford University Press.

MARCUSE, H. (1962), Eros and Civilization; A Philosophical Enquiry into Freud, New York, Vintage.

MARCUSE, H. (1968), Negations : Essays in Critical Theory, Translated by Jeremy J. Shapiro, Boston, Beacon Press.

MARCUSE, H. (1969), An Essay on Liberation, Boston, Beacon Press.

MATHIESEN, T. (1974), The Politics of Abolition - Scandinavian Studies in Criminology, vol. IV, London, Martin Robertson.

MELOSSI, D. (1975), Democracy and public order, Third conference of European Group for the Study of Deviance and Social Control, Amsterdan, 9.12 septembre.

MELOSSI, D. (1976), «The penal question in Capital», Crime and Social Justice, 5, 26-33. 
MELOSSI, D. (1977), Carcere e fabrica. Alle origini del sistema penitenziario. Bologne, Società editrice il Mullino.

MIAILlE, M. (1976), Une introduction critique au droit, Paris, Maspero.

MILLS, C.W. (1956), The Power Elite, New York, Oxford University Press.

MILLS, C.W. (1959), The Sociological Imagination, New York, Oxford University Press.

MILLS. C.W. (1960), «Letter to the New Left», New Left Review I (5), 18-23.

MILls, C.W. (1964), Sociology and Pragmatism: The Higher Learning in America, New York, Paine-Whitman.

MILLS, C.W. (édit.) (1962), The Marxists, New York, Dell.

PERALDI, F. (1976), «Institutions et appareils de pouvoir», Brèches, 3, (4).

PIRES, A.P. (1975), la Crise de la criminologie et les mouvements radicaux. Travail présenté dans le cadre du Séminaire de recyclage en criminologie, Université de Montréal, École de criminologie.

PLATT, A.M. (1970), «Saving and controlling delinquent youth - a critique», Issues in Criminology, 5, (1), 1-24.

PLATT, A.M. (1974), "Prospects for a radical criminology in the United States», Crime and Social Iustice, 1, 2-10.

QUINNEY, R. (1972), «The ideology of law : notes for a radical alternative to legal oppression», Issues in Criminology, 7, (1), 1-35.

QUINNEY, R. (1974), Critique of Legal Order, Boston, Little, Brown and Company.

ROBERT, Ph. (1972), la Sociologie entre une criminologie du passage à l'acte et une criminoloie de la réaction sociale. Service d'Études pénales et criminologiques, ministère de la Justice, France.

ROBERT, Ph. (1976), "Les statistiques criminelles ou l'histoire d'un contresens», Actes, 10, 7-17.

ROBERT, Ph. (1984), la question pénale, Paris, Droz.

SCHWENDINGER, H. et J. SCHWENDINGER (1970), «Defenders of order or guardians of human rights?», Issues in Criminology, 5, (2), 123-157.

SCHWENDINGER, H. et J. SCHWENDINGER (1972), «The continuing debate on the legalistic approach to the definition of crime», Issues in Criminology, 7, (1), 71-81.

SCHWENDINGER, H. et J. SCHWENDINGER (1974), The Sociologists of the Chair, New York, Basic Books.

SCHWENDINGER, H. et J. SCHWENDINGER (1977), «Social class and the definition of crime», Crime and Social Justice, 7, 4-13.

SZABO, D. (1977), «Histoire d'une expérience québécoise qui aurait pu mal tourner», Criminologie, 10 (2), 5-39.

TAKAGI, P. (1974), «A garrison state in a «democratic» society», Crime and Social Iustice, 1, 27-33.

TAYLOR, I. et L. TAYLOR (édit.) (1973), Politics and Deviance, Middlesex, Penguin Books.

TAYLOR, I., P. WALTON et J. YOUNG (1973), The New Criminology, London, Routledge and Kegan Paul.

TAYLOR, I., P. WALTON et J. YOUNG (édit.) (1975), Critical Criminology, London, Routledge and Kegan Paul. 
TURK, A.T. (1975), «Prospects and pitfalls for radical criminology : a critical response to Platt», Crime and Social Iustice, 4, 41-42.

VAN OUTRIVE, L. (1973), Sociologie de l'administration de la iustice, Notes de cours polycopiés, Université de Montréal, École de criminologie.

VAN OUTRIVE, L. (1977), «Interactionisme et néo-marxisme, une analyse critique», Déviance et Société, 1, (3), 253-291.

WELLMER, A. (1974), Critical Theory of Society, New York, The Seabury Press.

ZEITLIN, I.M. (1967), Marxism: A Re-examination, New York, Van Nostrand.

ZEITLIN, I.M. (1968), Ideology and the Development of Sociological Theory, Englewood Cliffs, N.J., Prentice-Hall. 